\title{
Insight into the structural requirement for Anticancer Activity: Pharmacophore Generation and 3D QSAR Analysis
}

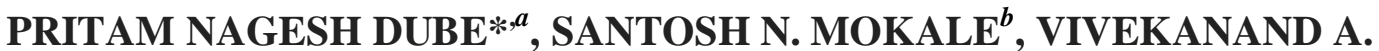 CHATPALLIWAR ${ }^{a}$}

\author{
*Corresponding Author: pritamdube@gmail.com \\ ${ }^{a}$ Department of Pharmaceutical Chemistry, Shri Neminath Jain Bhamhacharyashram's \\ Shreeman Sureshdada Jain College of Pharmacy, Chandwad, Nashik 423 101, Maharashtra, \\ India \\ ${ }^{b}$ Department of Pharmaceutical Chemistry, Y. B. Chavan College of Pharmacy, Aurangabad- \\ 431001, Maharashtra, India
}

\begin{abstract}
Transforming growth factor $\beta$ receptor-associated kinase 1 (TAK1) or mitogen activated-protein kinase kinase kinase 7 (MAP3K7) is a serine/threonine kinase which forms a key part of canonical immune and inflammatory signaling pathways. A 5-point pharmacophore model was developed and the generated pharmacophore model was used to derive a predictive atom-based 3D quantitative structure-activity relationship analysis (3D-QSAR) model for the studied dataset. The obtained 3D-QSAR model has an excellent correlation coefficient value $\left(\mathrm{r}^{2}=0.97\right)$ along with good statistical significance as shown by high Fisher ratio $(F=266.8)$. The QSAR model suggests that electron-withdrawing character, hydrogen bond-donating groups, hydrophobic and negative ionic groups positively contribute to the TAK1 inhibition.
\end{abstract}

Keyword: TAK1 inhibitors, anticancer, pharmacophore, 3D QSAR 


\section{Introduction}

Transforming growth factor $\beta$ receptor-associated kinase 1 (TAK1) or mitogen activated-protein kinase kinase kinase 7 (MAP3K7) is a serine/threonine kinase which forms a key part of canonical immune and inflammatory signaling pathways. ${ }^{1}$ TAK1 mediates signaling downstream of multiple cytokine receptors (TGF $\beta$ R, TNFR, TLRs, IL-1R, etc.). Although the precise mechanisms governing TAK1 activation and signaling are complex, involving extensive ubiquitination and the TAB family of adaptor proteins, it has been established that TAK1 directly phosphorylates and activates members of the MAP kinase kinase (MKK) family, including MKK3/4/6/7, as well as the IKK family, which are the immediate activators of the MAP kinases p38 and JNK and the transcription factor NFאB. ${ }^{2-4}$

Members of the family of nuclear factor $-\kappa \mathrm{B}(\mathrm{NF}-\kappa \mathrm{B})$ transcription factors regulate expression of a large number of genes involved in immune and inflammatory responses, as well as in cell survival, proliferation, and differentiation. Inappropriate activation of $\mathrm{NF}-\kappa \mathrm{B}$ signaling has been implicated in the pathogenesis of chronic inflammation, autoimmunity, and various cancers. ${ }^{5}$ For this reason, there has been some interest in exploring the therapeutic potential of TAK1 inhibitors in cancers with an inflammatory component, for example, ovarian and colorectal carcinomas, as well as in hematological malignancies. ${ }^{6-7}$

A large number of molecular modelling programs have been developed and widely used in the pharmaceutical and biological industry as well as in academia. The extensive applications of these software's and the chemical databases have made CADD a valuable tool in drug discovery and development process. ${ }^{8-10}$ Pharmacophore modelling involves extracting common chemical features (hydrogen-bond acceptors, hydrogen bond donors, hydrophobic regions and positively or negatively charged groups) from 3D structures of a set of known ligands, representative of essential ligand-receptor interactions. In medicinal chemistry, pharmacophore modelling is used not only for hit-and-lead identification but also for lead optimization, drug design and discovery. 3D QSAR analysis is performed for generating models which correlates biological activity with physico-chemical properties of the molecules. A statistically significant 3D QSAR model helps in better understanding of structure activity relationship of a series of molecules and predicts the activity of yet to be synthesized compounds. ${ }^{11}$

Related to the foregoing studies, the present paper reports 3D-QSAR analysis of set of 7aminofuro [2,3-c]pyridine derivatives, reported by Hornberger K. R. et al. ${ }^{12}$ and intends to 
provide the platform to develop new compounds over existing substituted pyridines. Pyridines scaffolds are to modify and a range of molecules can be synthesized. However, substituted pyridines exhibit a range of diversity in the structures as well as in biological activities so this series of molecules is good for generating the significant quantitative relationship. The developed atom-based 3D-QSAR model highlight the structural features of pyridine analogs for binding to TAK-1 which is useful for further design of more potent TAK-1 inhibitory agent having anticancer activity.

\section{Materials and Methods}

\subsection{Dataset for analysis}

The present 3D-QSAR study was performed on substituted 7-aminofuro [2,3-c]pyridine derivatives synthesized by Hornberger K. R. et al. ${ }^{12}$ for the development of ligand based CPHs (common pharmacophore hypothesis). Out of 57 compounds, 54 compounds with well defined TAK-1 inhibitory activity (given as $\mathrm{IC}_{50}$ values in $\mu \mathrm{M}$ concentration) were used for the present investigation. The compounds 12n, 12p and 12au were omitted from the QSAR set due to their improper activity. The biological activities, $\mathrm{IC}_{50}$, were transformed into $\mathrm{pIC}_{50}=\left[-\log \mathrm{IC}_{50}\right]$ (Table 1) and used as dependent variable for 3D-QSAR model generation. The dataset consists of both active and inactive molecules, and the dataset was divided into training and test set using the "Automated Random Selection", option present in the PHASE software. The total set of inhibitors was divided randomly into a training set (35 compounds) for generation of 3D QSAR models and a test set (19 compounds) for validation of the developed model. The partitioning was so selected that there should be both active and inactive ligands in each test as well as training set. The dataset was then used for generating common pharmacophore hypotheses and subsequently for developing 3D-QSAR models (Tables 1).

\subsection{Computational details}

The 3D-QSAR pharmacophore model developing study was performed using the PHASE 3.4 module of Schrodinger molecular modeling software. ${ }^{13}$ PHASE is a versatile product to identify common pharmacophore hypothesis, which is accompanied by a set of aligned conformations that suggests the relative manner in which the molecules are likely to bind to the receptor. A given hypothesis gives 3D-QSAR model to predict activity. The pharmacophore model was 
developed using a set of pharmacophore features to generate sites for all the compounds. Each structure is represented by various chemical features that may make easy non-covalent binding among the ligand and its binding pocket. ${ }^{14,15}$

LigPrep provides a structure cleaning step, ${ }^{16}$ which is incorporated into PHASE. ${ }^{17}$, PHASE provides two built-in approaches for the purposes of pharmacophore model development, both of which employ the MacroModel conformational search engine. Conformational analysis were performed using Monte- Carlo Multiple Minimum method implemented in the Schrodinger software. $^{13}$

The ligands were assigned as active (above 6.2) and inactive (below 5.7) by giving an appropriate activity threshold value. The activity threshold value was selected on the basis of dataset activity distribution (4.590-8.398) and the active ligands were chosen to derive a set of suitable pharmacophores. Common pharmacophore and QSAR model building were generated by prepared ligands.

\subsection{Scoring pharmacophores with respect to active and inactive ligands}

The resulting pharmacophores were then scored and ranked. The overall ranking of all the hypotheses based on scored hypothesis. The scoring algorithm included the contributions from the alignment of site points and vectors, volume overlap, selectivity, number of ligands matched, relative conformational energy, and activity.

\subsection{Perceiving common pharmacophores}

Common pharmacophores are examined by a scoring protocol to identify the pharmacophore from each surviving n-dimensional box that yields the best alignment of the active set ligands. The scoring protocol provides a ranking of different hypotheses to choose most appropriate for further investigation. Also the inactive molecules were scored in order to observe the alignment of these molecules with respect to the different pharmacophore hypotheses and to select the best ones. The larger is the difference between the score of active and inactives, the better is the hypothesis at discriminating the active from inactive molecules. 


\subsection{Building 3D-QSAR models}

3D-QSAR models represented by binary-valued occupation patterns that can be used as independent variables to create partial least-squares (PLS) factors. Statistics on the correlation of predicted with actual activity data were collated for the hypothesis.

PHASE QSAR models may be either atom-based or pharmacophore- based, the difference being whether all atoms are taken into account, or merely the pharmacophore sites that can be matched to the hypothesis. Pharmacophore- based QSAR models were generated for hypothesis using a grid spacing of $1.0 \AA$ and the 35 -member training set. QSAR models were validated by predicting the activity of test set ligands containing one to four PLS factors.

\section{Results and Discussions}

\subsection{Generation of 3D-QSAR models}

Different variant $\mathrm{CPHs}$ were generated by common pharmacophore identification process. All CPHs were examined and scored to identify the pharmacophore that yields the best alignment of the active compounds $\left(\mathrm{pIC}_{50}>6.2\right)$. All CPHs were validated by aligning and scoring the inactive compounds $\left(\mathrm{pIC}_{50}<5.7\right)$. We have selected top two $\mathrm{CPHs}$ models whose survival-inactive scores ranked in the top $1 \%$ for alignment of all compounds and 3D-QSAR studies. The survival score for these CPHs is shown in Table 2. All top two CPHs were found to be associated with the fivepoint hypotheses, which consists of one hydrogen bond acceptor (A), one hydrogen bond donor (D), one hydrophobic $(\mathrm{H})$, and two aromatic rings $(\mathrm{R})$ vector features. All compounds were aligned using CPH ADHRR.84 for 3D-QSAR study (Fig. 1) alignment of active compounds by using CPH ADHRR.651 was shown in Fig. 2.

All top CPHs were used for atom-based 3D-QSAR model generation. The CPH ADHRR.84 yielded a 3D-QSAR model with good value of regression coefficient, low standard deviation, and high variance ratio with good stability, but showed diminished predictive power along with high RMSE value and low Pearson R value which stand for correlation between predicted and observed activity for test set. The CPHs ADHRR.84 and ADHRR.651 yielded 3D-QSAR models with good PLS statistical values. Both these hypotheses showed good internal as well as external predictive power (Table $3 \& 4$ ). The training set correlation in both CPHs is characterized by PLS factors $\left(\mathrm{R}^{2}=0.9612, \mathrm{SD}=0.1705, \mathrm{~F}=185.9, \mathrm{P}=1.043 \mathrm{e}-020, \mathrm{Q}^{2}=0.7949\right.$ for $\mathrm{CPH}$ 
ADHRR.84 and $\mathrm{R}^{2}=0.9727, \mathrm{SD}=0.1431, \mathrm{~F}=266.8, \mathrm{P}=5.552 \mathrm{e}-023, \mathrm{Q}^{2}=0.8051$ for $\mathrm{CPH}$ ADHRR.651).

We have selected the 3D-QSAR models generated by CPHs ADHRR.84 and ADHRR.651 for correlating the structure with activity. Graph of observed versus predicted biological activity of training and test sets are shown in Figs. 3 and 4, respectively. Residuals values obtained by subtraction of predicted activities from observed biological activities are near to zero which indicated that error in prediction of biological activity is low and predicting ability of QSAR models developed by CPHs ADHRR.84 and ADHRR.651 is good (Table 5). Mean of residual in predicting the activity of compounds was calculated by average of summation of all residual values. 3D-QSAR models associated with hypotheses ADHRR.84 and ADHRR.651 showed 0.1828 and 0.1554 , respectively, as the mean of residual which also support the predictability of both QSAR models. The pharmacophore hypothesis showing distance between pharmacophoric sites is depicted in Fig. 5.

Based on overall statistical results, 3D-QSAR model developed using CPHs ADHRR.84 and ADHRR.651 was applied to each compound in the series for the establishment of structureactivity correlation. A pictorial representation of the cubes generated in the present 3D-QSAR along with most active and inactive compounds is shown in Figs. 6 and 7. In these generated cubes, the blue cubes indicate favorable features, while red cubes indicate unfavorable features for biological activity. The blue cubes around the hydrogen bond acceptor and hydrogen bond donor group of thiophene ring suggest the importance of core ring for activity.

\subsection{SAR analysis by visualization of regression coefficients}

Phase allows us to visualize effects due to each pharmacophoric feature and the positive and negative regression coefficients associated with the effect, like $\mathrm{H}$-bond donor effects, hydrophobic effects, negative ionic effects, positive ionic effects and electron-withdrawing effects. 3D-QSAR model visualization in Phase depends on the module used. In the grid-based 3D-QSAR method used here, visualization of the model is referred to as "regression coefficient visualization" or "effects" from atom or pharmacophore types. In field-based QSAR methods, the results are represented by field "contours". In this study we looked at effects of each pharmacophoric feature - H-bond donor, hydrophobic, negative ion and positive ion - with their

positive and negative regression coefficients around the pharmacophore hypothesis to visualize 
the favorable and unfavorable regions. In all analyses, positive coefficients and negative coefficients are shown as blue-colored and red-colored cubes, respectively.

\subsubsection{Analysis of hydrogen bond donor effects}

Ligand-receptor interactions inferred by the 3D-QSAR analysis can be visualized as color-coded cubes in the space around the ligand, for each of the properties considered. For the convenience and ease of viewing, the hydrogen-bond donor effects are visualized at a positive regression coefficient threshold of 0.008 and a negative regression coefficient threshold of -0.008 . The presence of hydroxyl group on $3^{\text {rd }}$ position (blue-colored region) in the active compound 12ad acts as a hydrogen bond donor and that might be a reason for high activity of the compound, whereas absence of hydrogen bond donor group at same position leads to decrease in activity of inactive compounds 12ag and 12ao.

\subsubsection{Analysis of hydrophobic effects}

Hydrophobic effects are visualized at a positive and a negative regression coefficient threshold of 0.040 and -0.040 , respectively. Blue colored cubes at thiophene ring in compound 12az indicate the importance of hydrophobic groups at that position and the absence of hydrophobic groups at this position might lead to a decrease in activity. Addition of various hydrophobic

groups at $3^{\text {rd }}$ and $4^{\text {th }}$ position (red-color) of substituted benzene ring results in decrease in activity of compounds can be observed by considering 12a (5.9), 12ae (5.7), 12ai (5.5) and 12as (5.4). Active compound that gives rise to strong and positive hydrophobic effects are shown in Fig. 6a and $7 \mathrm{a}$.

\section{Conclusion}

Computational methods have become increasingly important in a number of areas such as comparative or homology modelling, functional site location, characterization of ligand-binding sites in proteins, docking of small molecules into protein binding sites, protein-protein docking, and molecular dynamics simulations. The results obtained yield information that sometimes is beyond current experimental possibilities and can be used to guide and improve a vast array of experiments. On the basis of our improved level of understanding of molecular recognition and the widespread availability of target structures, it is reasonable to assume that computational 
methods will continue aiding not only in the design and interpretation of hypothesis-driven experiments in the field of cancer research but also in the rapid generation of new hypotheses.

The two 3D-QSAR models were developed using variant CPHs which consist of one acceptor, one donor, one hydrophobic, and two rings vector feature. This 3D-QSAR model gives a hypothetical image for designing of new potential compounds. The overall study provides detailed structure and important binding information of pyrimidine derivatives as TAK1 inhibitors for anticancer activity.

\section{Acknowledgments}

The authors wish to acknowledge the Department of Science and Technology, India for financial support to carry out this work. 


\section{References}

1. Walczak H, Miller RE, Ariail K. Tumoricidal activity of tumor necrosis factor-related apoptosis-inducing ligand. Nat Med 1999;5:157-163.

2. Cretney E, Shanker A, Yagita H, Smyth MJ, Sayers TJ. TNF-related apoptosisinducing ligand as a therapeutic agent in autoimmunity and cancer. Immunol Cell Biol 2006;84:87-98.

3. Sakurai H, Shigemori N, Hasegawa K, Sugita T. TGF- $\beta$-activated kinase 1 stimulates $\mathrm{NF}-\kappa \mathrm{B}$ activation by an NF- $\mathrm{BB}$-inducing kinase independent mechanism. Biochem Biophys Res Commun 1998;243:545-549.

4. Sakurai H, Miyoshi H, Toriumi W, Sugita T. Functional interactions of transforming

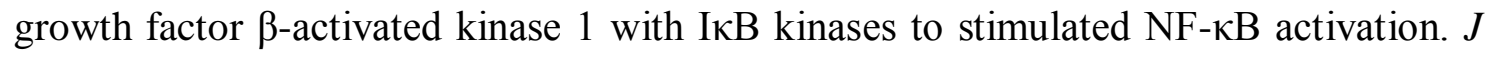
Biol Chem 1999;274:10641-10648.

5. Wiley SR, Schooley K, Smolak PJ. Identification and characterization of a new member of the TNF family that induces apoptosis. Immunity 1995;3:673-682.

6. Chaoo MK, Sakurai H, Koizumi K, Saiki I. TAK1-mediated stress signaling pathways are essential for TNF- $\alpha$-promoted pulmonary metastasis of murine colon cancer cells. Int J Cancer 2006;118:2758-2764.

7. Sato S, Sanjo H, Takeda K. Essential function for the kinase TAK1 in innate and adaptive immune responses. Nat Immunol 2005;6:1087-1095.

8. Lokwani DK, Sarkate AP, Shinde DB. 3D-QSAR and docking studies of benzoyl urea derivatives as tubulin-binding agents for antiproliferative activity. Med Chem Res 2013;22:1415-1425.

9. Kristam R, Parmar V, Viswanadhan VN. 3D-QSAR analysis of TRPV1 inhibitors reveals a pharmacophore applicable to diverse scaffolds and clinical candidates. $J$ Mol Graph Model 2013;45:157-172.

10. Tanwar O, Marella A, Shrivastava S, Alam MM, Akhtar M. Pharmacophore model generation and 3D-QSAR analysis of $\mathrm{N}$-acyl and $\mathrm{N}$-aroylpyrazolines for enzymatic and cellular B-Raf kinase inhibition. Med Chem Res 2013;22:2174-2187.

11. Lokwani D, Shah R, Mokale S, Shastry P, Shinde D. Development of energetic pharmacophore for the designing of 1,2,3,4-tetrahydropyrimidine derivatives as selective cyclooxygenase-2 inhibitors. J Comput Aided Mol Des 2012;26:267-277. 
12. Hornberger KR, Berger DM, Crew AP, Dong H, Kleinberg A, Li A, Medeiros MR, Mulvihill MJ, Siu K, Tarrant J, Wang J, Weng F, Wilde VL, Albertella M, Bittner M, Cooke A, Gray MJ, Maresca P, May E, Meyn P, Peick W, Romashko D, Tanowitz M, Tokar B. Discovery and optimization of 7-aminofuro[2,3-c]pyridine inhibitors of TAK1. Bioorg Med Chem Lett 2013;23:4517-4522.

13. Dixon SL, Smondyrev AM, Knoll EH, Rao SN, Shaw DE, Friesner RA. PHASE: a new engine for pharmacophore perception, 3D QSAR model development, and 3D database screening:1. Methodology and preliminary result. J Comput Aided Mol Des 2006;20:647-671.

14. Dixon SL, Smondyrev AM, Rao S. PHASE: A Novel Approach to Pharmacophore Modeling and 3D Database Searching. Chem Biol Drug Des 2006;67:370-372.

15. Dube PN, Mokale S, Datar P. CoMFA and Docking Study of 2,N6-disubstituted 1,2dihydro-1,3,5-triazine-4,6-diamines as Novel PfDHFR Enzyme Inhibitors for antimalarial activity. Bulletin Facult Pharmacy Cairo Univ 2014;52:125-134.

16. LigPrep, Version 2.5, Schrodinger, 2009, LLC, New York.

17. PHASE-3.4, Schrodinger, 2009, LLC New York. 


\section{Figure citation}

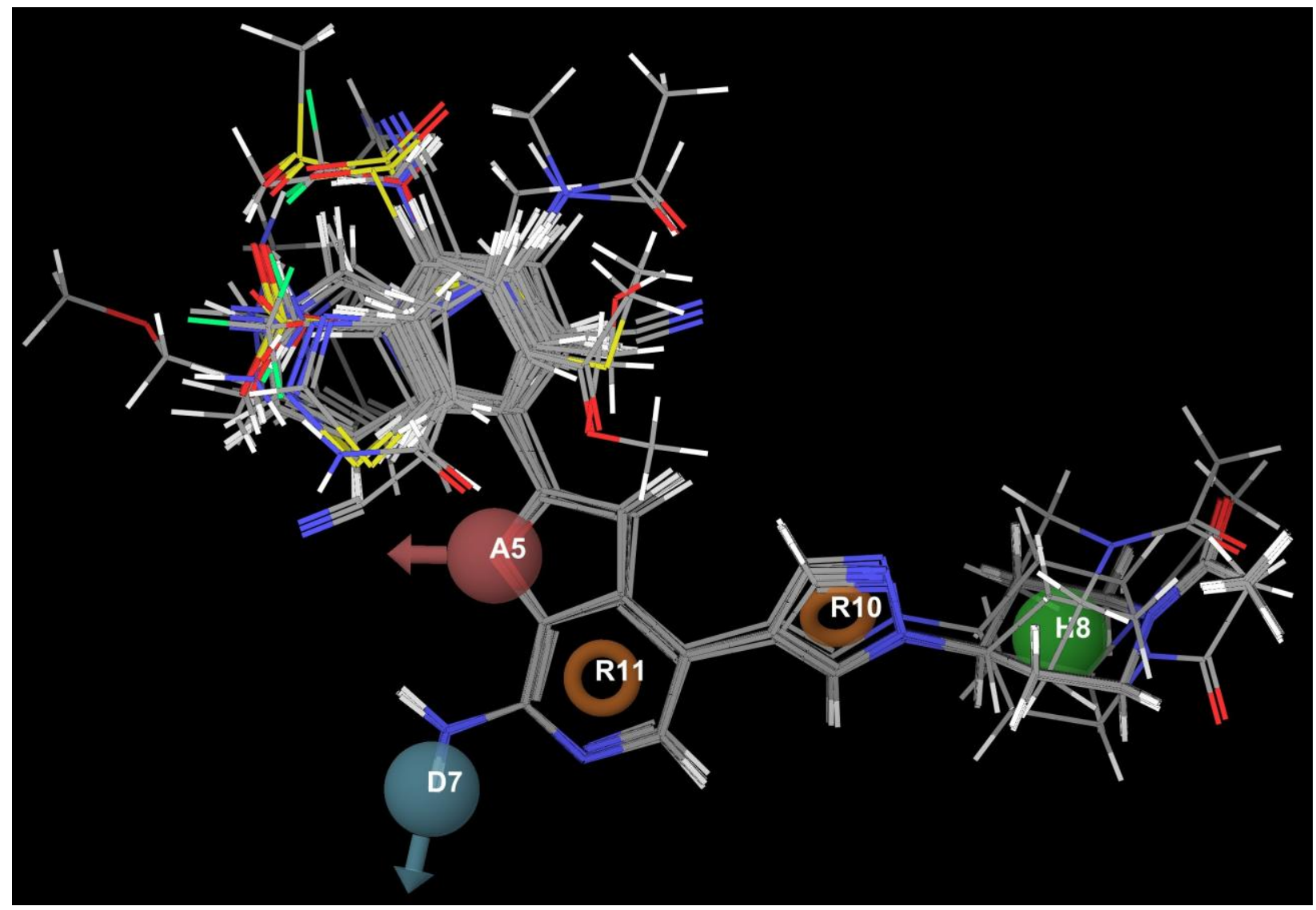

Figure 1: Alignment of all compounds using the 5-point pharmacophore hypothesis $\mathrm{CPH}$ ADHRR.84 


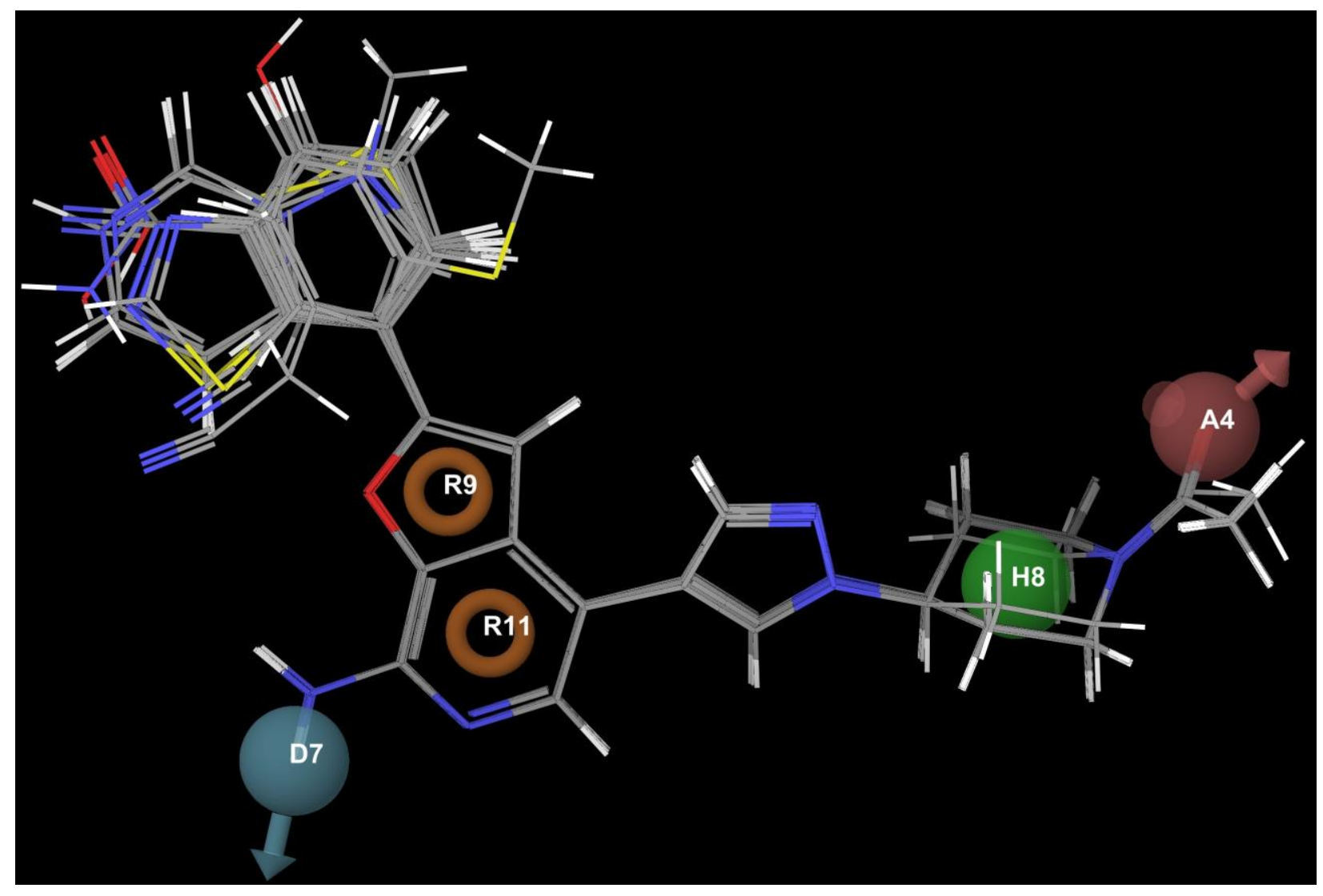

Figure 2: Alignment of active compounds using the 5-point pharmacophore hypothesis $\mathrm{CPH}$ ADHRR.651
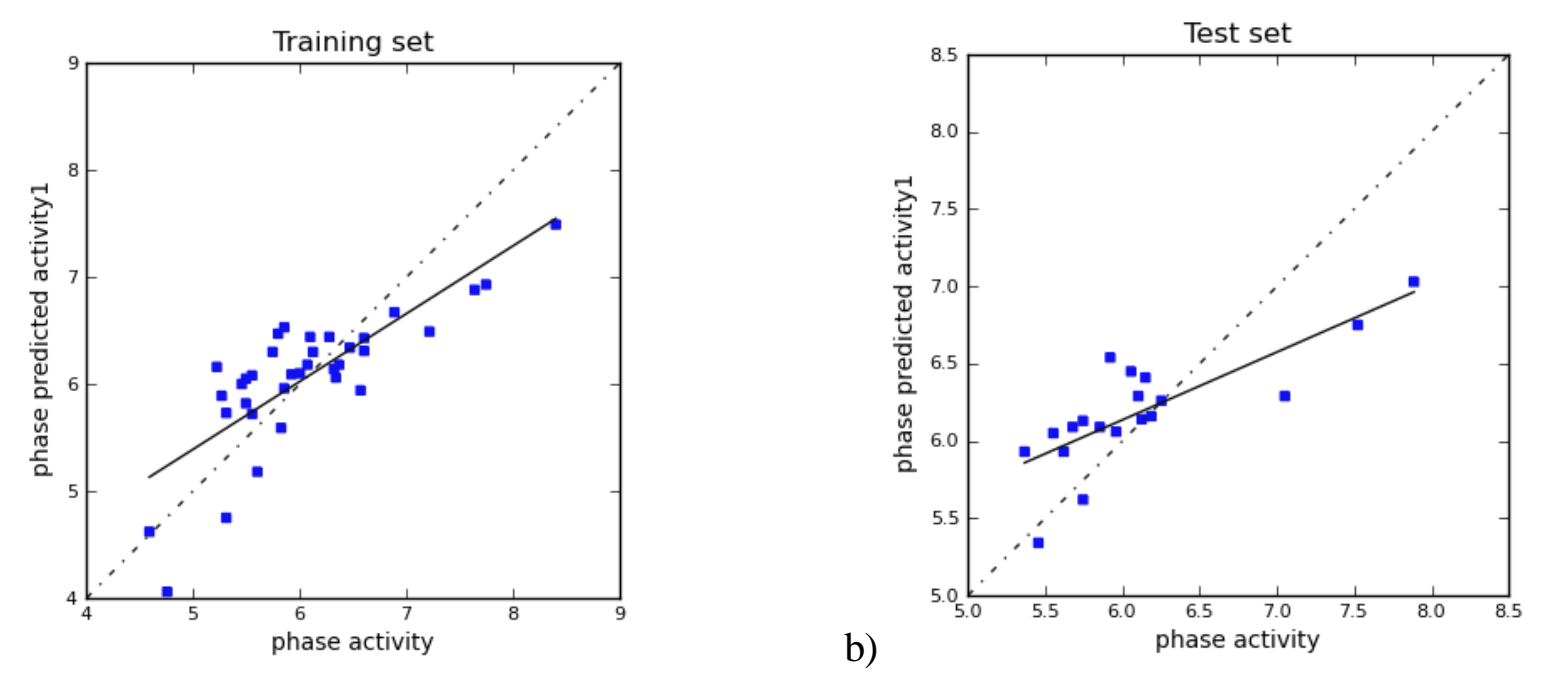

Figure 3: Plot of experimental versus predicted $\mathrm{pIC}_{50}$ values of compounds for the 3D-QSAR model CPH ADHRR.84 a) Training set \& b) Test set 

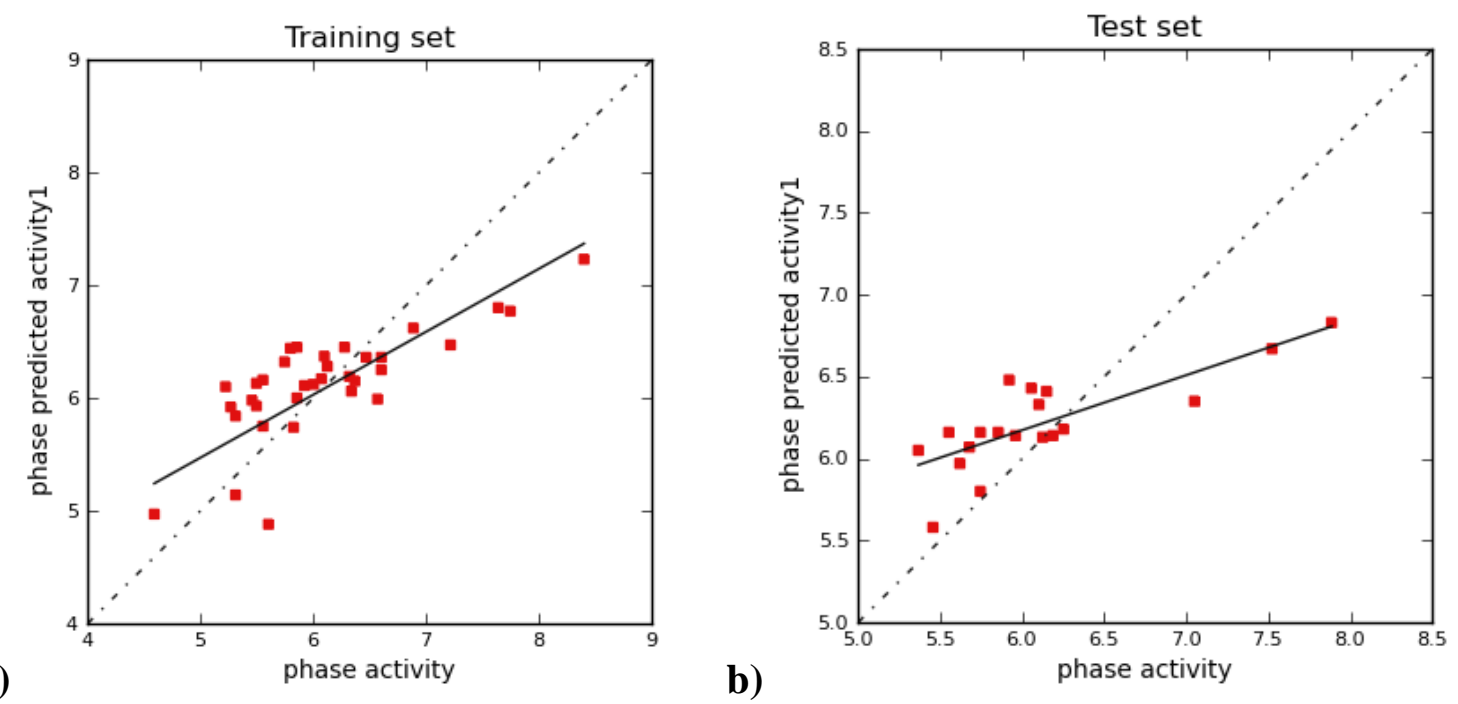

Figure 4: Plot of experimental versus predicted $\mathrm{pIC}_{50}$ values of compounds for the 3D-QSAR model CPH ADHRR.651 a) Training set \& b) Test set

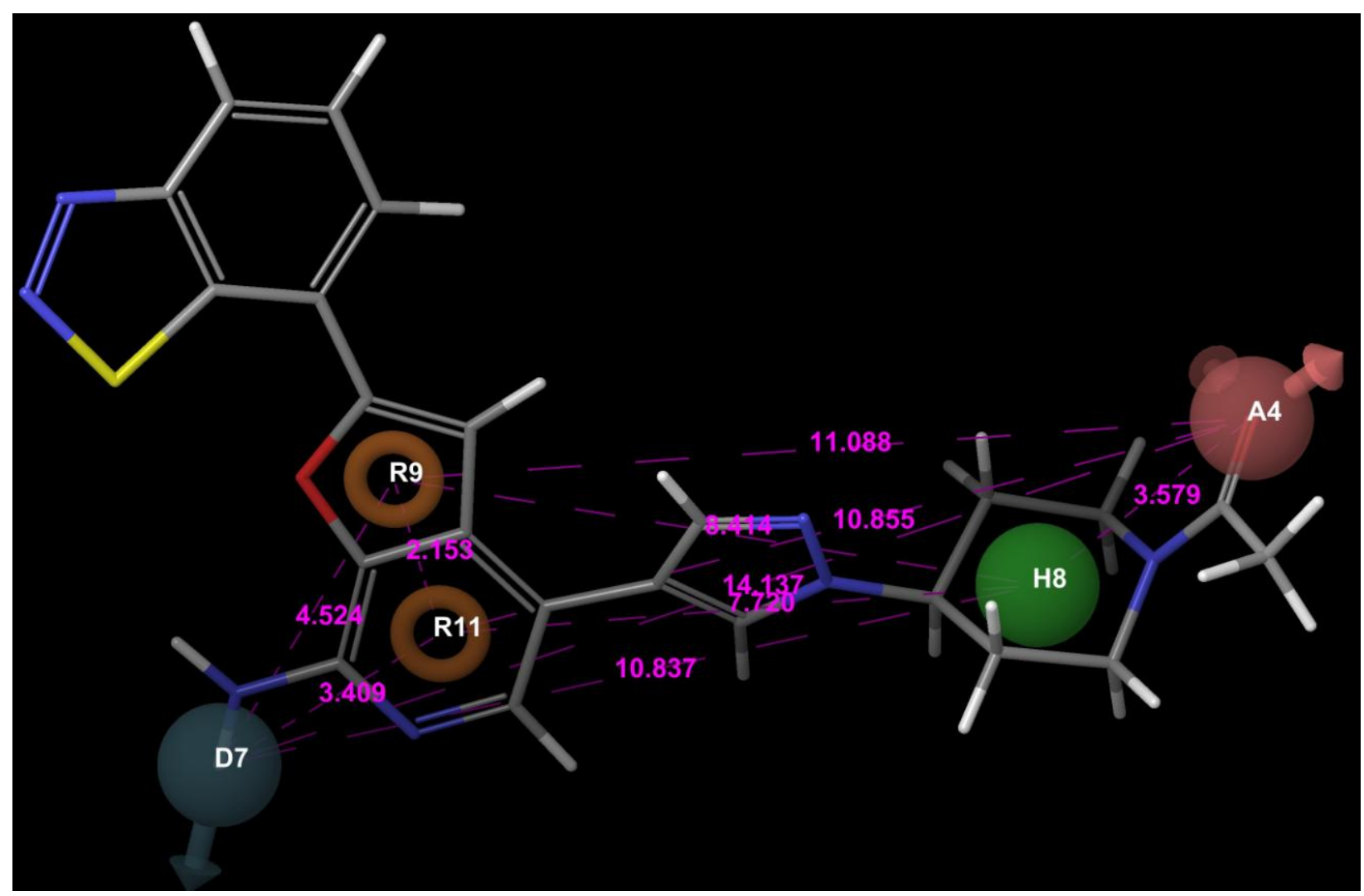

Figure 5: The 5-point pharmacophore hypothesis that gave the best 3D-QSAR model; Green represents hydrophobic feature, cyan represents hydrogen-bond donor and brown-colored ring represents a ring aromatic; All the inter-feature distances are in Angstrom $(\AA)$. 

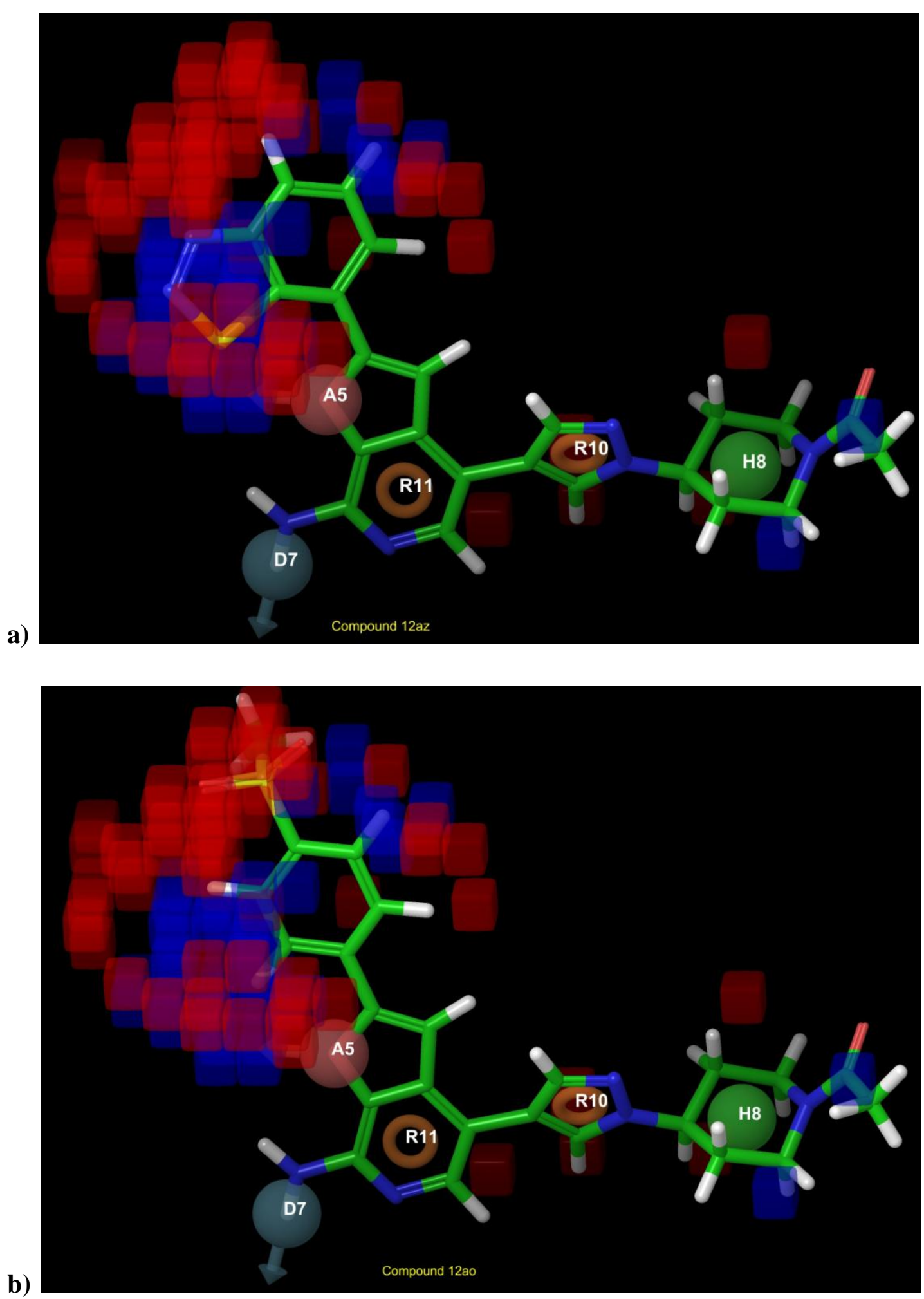

Figure 6: Pictorial representation of the cubes generated using the QSAR model developed using CPH ADDHR.84 for a) most active compound 12az and b) least active compound 12ao. 
Blue cubes indicate favorable regions, while red cubes indicate unfavorable region for the activity.

a)

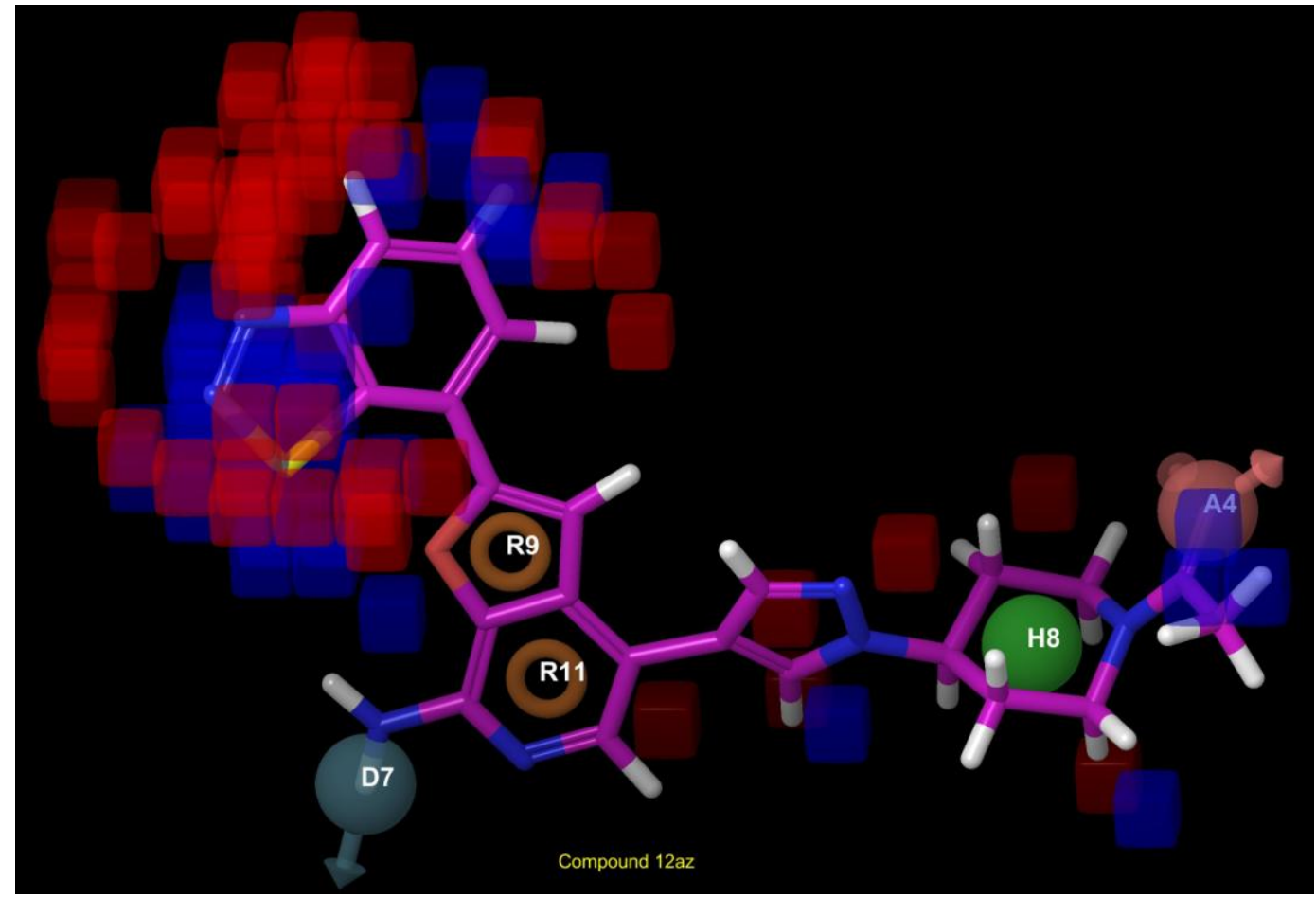

b)

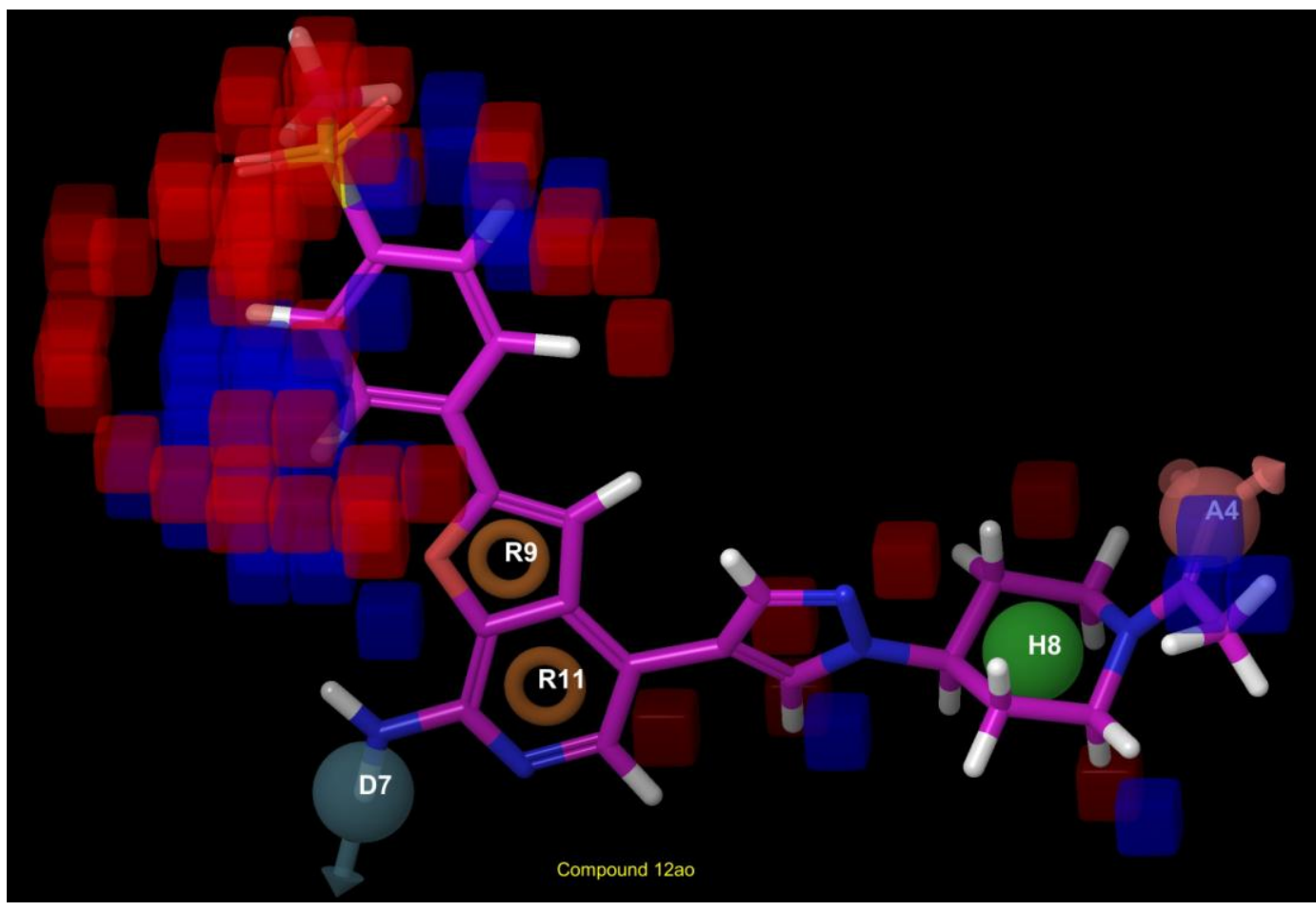

Figure 7: Pictorial representation of the cubes generated using the QSAR model developed using CPH ADDHR.84 for a) most active compound 12az and b) least active compound 12ao. 
Table 1: Structure of compounds used for development of common pharmacophore hypothesis and 3D-QSAR studies along with biological activity

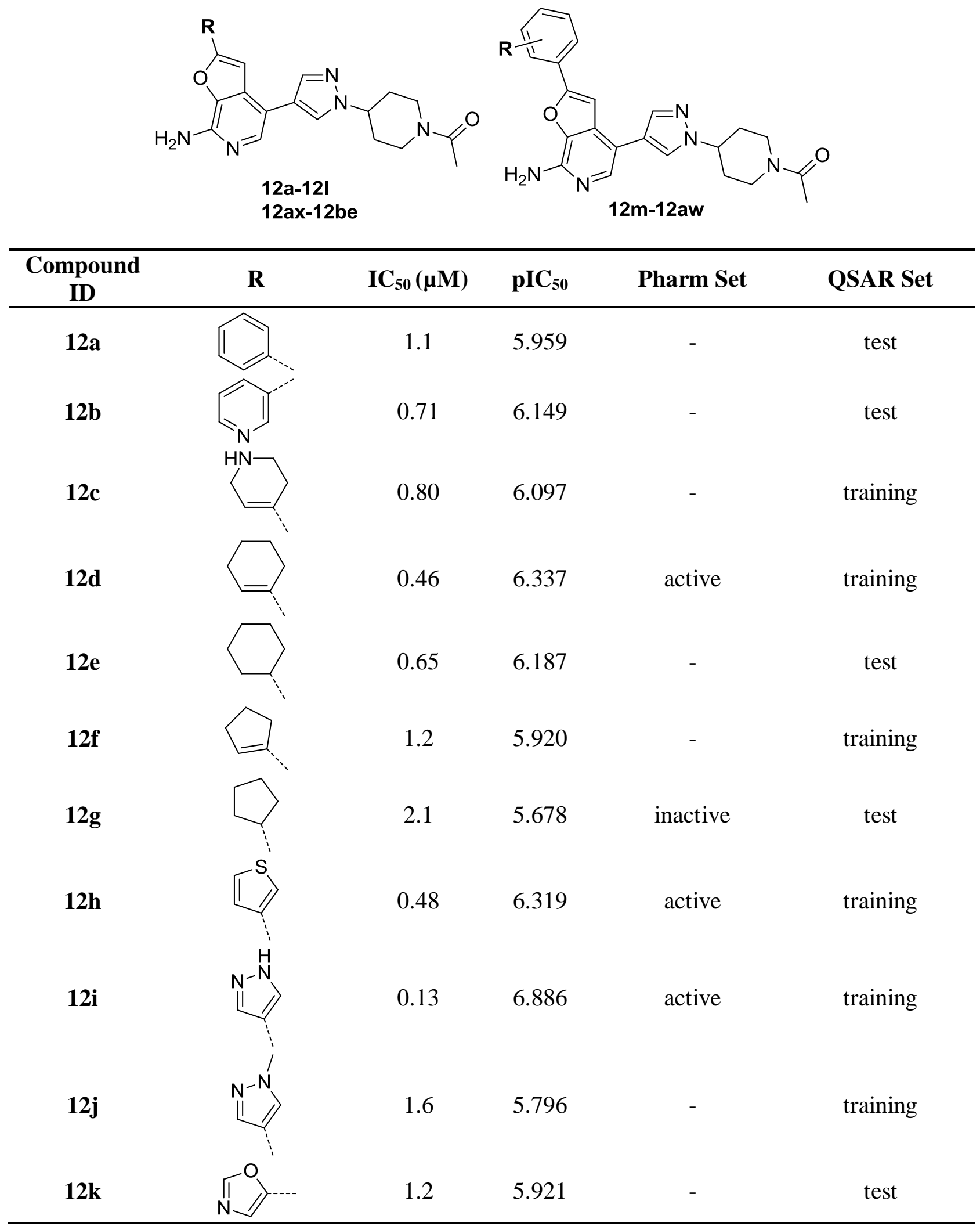




\begin{tabular}{|c|c|c|c|c|c|}
\hline 121 & $\stackrel{N-S}{N-S}$ & 0.88 & 6.055 & - & test \\
\hline $12 \mathrm{~m}$ & $\mathrm{H}$ & 0.43 & 6.366 & active & training \\
\hline $12 n *$ & 2-SMe & $>30$ & $<4.523$ & - & NA \\
\hline 120 & $2-\mathrm{CN}$ & 0.56 & 6.2518 & active & test \\
\hline $12 p *$ & $2-\mathrm{CF}_{3}$ & $>30$ & $<4.523$ & - & NA \\
\hline $12 q$ & 2-COMe & 5.4 & 5.268 & inactive & training \\
\hline $12 r$ & $2-\mathrm{CONH}_{2}$ & 6.0 & 5.229 & inactive & training \\
\hline $12 s$ & $2-\mathrm{CH}_{2} \mathrm{OH}$ & 0.75 & 6.125 & - & test \\
\hline $12 t$ & $2-\mathrm{CH}_{2} \mathrm{OMe}$ & 1.4 & 5.854 & - & training \\
\hline $12 u$ & $2-\mathrm{CH}_{2} \mathrm{CN}$ & 0.25 & 6.602 & active & training \\
\hline $12 \mathrm{v}$ & 3-SMe & 4.3 & 5.366 & inactive & test \\
\hline $12 w$ & $3-\mathrm{SO}_{2} \mathrm{Me}$ & 4.9 & 5.310 & inactive & training \\
\hline $12 x$ & $3-\mathrm{SO}_{2} \mathrm{NH}_{2}$ & 1.8 & 5.745 & - & test \\
\hline $12 y$ & $3-\mathrm{SO}_{2} \mathrm{NMe}_{2}$ & 1.4 & 5.854 & - & test \\
\hline $12 z$ & $3-\mathrm{NO}_{2}$ & 0.089 & 7.051 & active & test \\
\hline $12 \mathbf{a a}$ & $3-\mathrm{NH}_{2}$ & 0.77 & 6.113 & - & training \\
\hline $12 \mathbf{a b}$ & $3-\mathrm{NMe}_{2}$ & 2.4 & 5.620 & inactive & test \\
\hline $12 \mathrm{ac}$ & 3-NHCOMe & 3.2 & 5.495 & inactive & training \\
\hline $12 \mathrm{ad}$ & $3-\mathrm{OH}$ & 0.061 & 7.215 & active & training \\
\hline $12 \mathbf{a e}$ & 3-OMe & 1.8 & 5.745 & - & training \\
\hline 12af & $3-\mathrm{O}\left(\mathrm{CH}_{2}\right)_{2} \mathrm{OMe}$ & 3.2 & 5.495 & inactive & training \\
\hline $12 \mathrm{ag}$ & $3-\mathrm{OCF}_{3}$ & 3.5 & 5.456 & inactive & training \\
\hline 12ah & $3-\mathrm{CN}$ & 0.25 & 6.602 & active & training \\
\hline $12 \mathbf{a i}$ & $3-\mathrm{CF}_{3}$ & 2.8 & 5.553 & inactive & test \\
\hline 12aj & 3-COMe & 2.8 & 5.553 & inactive & training \\
\hline 12ak & $3-\mathrm{CONH}_{2}$ & 0.53 & 6.276 & active & training \\
\hline 12al & $3-\mathrm{CH}_{2} \mathrm{NH}_{2}$ & 0.79 & 6.102 & - & test \\
\hline 12am & $3-\mathrm{CH}_{2} \mathrm{OH}$ & 0.85 & 6.070 & - & training \\
\hline
\end{tabular}




\begin{tabular}{|c|c|c|c|c|c|}
\hline 12an & 4-SMe & 17.5 & 4.757 & inactive & training \\
\hline $12 \mathrm{ao}$ & $4-\mathrm{SO}_{2} \mathrm{Me}$ & 25.7 & 4.590 & inactive & training \\
\hline 12ap & $4-\mathrm{NH}_{2}$ & 1.8 & 5.745 & - & test \\
\hline $12 \mathrm{aq}$ & $4-\mathrm{NHSO}_{2} \mathrm{Me}$ & 4.9 & 5.310 & inactive & training \\
\hline 12 ar & $4-\mathrm{OH}$ & 0.27 & 6.569 & active & training \\
\hline 12 as & $4-\mathrm{OCF}_{3}$ & 3.5 & 5.456 & inactive & test \\
\hline 12at & $4-\mathrm{CN}$ & 2.5 & 5.602 & inactive & training \\
\hline $12 \mathbf{a u} *$ & 4-CONMe 2 & $>30$ & $<4.523$ & - & NA \\
\hline $12 \mathrm{av}$ & $4-\mathrm{CH}_{2} \mathrm{OH}$ & 2.8 & 5.553 & inactive & training \\
\hline $12 \mathrm{aw}$ & $4-\mathrm{CH}_{2} \mathrm{CN}$ & 1.5 & 5.824 & - & training \\
\hline $12 \mathrm{ax}$ & & 0.023 & 7.638 & active & training \\
\hline 12ay & & 0.013 & 7.886 & active & test \\
\hline $12 \mathbf{a z}$ & & 0.004 & 8.398 & active & training \\
\hline $12 \mathrm{ba}$ & & 0.030 & 7.523 & active & test \\
\hline $12 \mathrm{bb}$ & & 0.018 & 7.745 & active & training \\
\hline $12 b c$ & & 1.0 & 6.000 & - & training \\
\hline $12 \mathrm{bd}$ & & 0.34 & 6.468 & active & training \\
\hline 12be & & 1.4 & 5.854 & - & training \\
\hline
\end{tabular}

*omitted from QSAR analysis 
Table 2: Score of different parameters of the hypothesis ADHRR-84 and ADHRR-651

\begin{tabular}{cccc}
\hline & & \multicolumn{2}{c}{ Score } \\
\cline { 3 - 4 } Sr. No. & Parameter & ADHRR-84 & ADHRR-651 \\
\hline 1. & Survival & 3.880 & 3.864 \\
2. & Survival-inactive & 1.041 & 1.056 \\
3. & Post hoc & 5.860 & 5.844 \\
4. & Site & 0.97 & 0.95 \\
5. & Vector & 1.000 & 0.999 \\
6. & Volume & 0.908 & 0.911 \\
7. & Selectivity & 1.869 & 1.971 \\
8. & Matches & 17 & 17 \\
9. & Energy & 0.00 & 17 \\
10. & Activity & 6.602 & 6.602 \\
11. & Inactive & 2.838 & 2.808 \\
\hline
\end{tabular}


Table 3: 3D-QSAR statistical parameters for ADHRR-84 hypothesis

\begin{tabular}{cccccccc}
\hline PLS factors & SD & $\mathbf{r}^{\mathbf{2}}$ & $\mathbf{F}$ & $\mathbf{P}$ & $\mathbf{R M S E}$ & $\mathbf{q}^{\mathbf{2}}$ & Pearson-R \\
\hline $\mathbf{1}$ & 0.4993 & 0.6342 & 57.2 & $1.059 \mathrm{e}-008$ & 0.4367 & 0.5568 & 0.7895 \\
$\mathbf{2}$ & 0.3043 & 0.8682 & 105.4 & $8.297 \mathrm{e}-015$ & 0.4071 & 0.6146 & 0.8027 \\
$\mathbf{3}$ & 0.2168 & 0.9352 & 149.1 & $1.679 \mathrm{e}-018$ & 0.3423 & 0.7276 & 0.8684 \\
$\mathbf{4}$ & $\mathbf{0 . 1 7 0 5}$ & $\mathbf{0 . 9 6 1 2}$ & $\mathbf{1 8 5 . 9}$ & $\mathbf{1 . 0 4 3 e - 0 2 0}$ & $\mathbf{0 . 2 9 7}$ & $\mathbf{0 . 7 9 4 9}$ & $\mathbf{0 . 9 0 9 3}$ \\
\hline
\end{tabular}

Bold font signifies the best hypothesis

Table 4: 3D-QSAR statistical parameters for ADHRR-651 hypothesis

\begin{tabular}{cccccccc}
\hline PLS factors & $\mathbf{S D}$ & $\mathbf{r}^{\mathbf{2}}$ & $\mathbf{F}$ & $\mathbf{P}$ & $\mathbf{R M S E}$ & $\mathbf{q}^{2}$ & Pearson-R \\
\hline $\mathbf{1}$ & 0.5489 & 0.5578 & 41.6 & $2.569 \mathrm{e}-007$ & 0.4776 & 0.4697 & 0.7878 \\
$\mathbf{2}$ & 0.3230 & 0.8515 & 91.8 & $5.566 \mathrm{e}-014$ & 0.3918 & 0.6431 & 0.8247 \\
$\mathbf{3}$ & 0.2004 & 0.9446 & 176.3 & $1.463 \mathrm{e}-019$ & 0.3436 & 0.7256 & 0.8884 \\
$\mathbf{4}$ & $\mathbf{0 . 1 4 3 1}$ & $\mathbf{0 . 9 7 2 7}$ & $\mathbf{2 6 6 . 8}$ & $\mathbf{5 . 5 5 2 e - 0 2 3}$ & $\mathbf{0 . 2 8 9 5}$ & $\mathbf{0 . 8 0 5 1}$ & $\mathbf{0 . 9 2 5 8}$ \\
\hline
\end{tabular}

Bold font signifies the best hypothesis 
Table 5: Comparison of observed biological activity and predicted activity along with fitness of compounds on CPHs

\begin{tabular}{|c|c|c|c|c|c|c|c|}
\hline \multirow{2}{*}{$\begin{array}{c}\text { Compound } \\
\text { ID }\end{array}$} & \multirow{2}{*}{$\begin{array}{l}\text { Observed } \\
\text { activity }\end{array}$} & \multicolumn{3}{|c|}{ ADHRR-84 } & \multicolumn{3}{|c|}{ ADHRR-651 } \\
\hline & & $\begin{array}{c}\text { Predicted } \\
\text { activity }\end{array}$ & Residual & $\begin{array}{c}\text { Fitness } \\
\text { score }\end{array}$ & $\begin{array}{c}\text { Predicted } \\
\text { activity }\end{array}$ & Residual & $\begin{array}{c}\text { Fitness } \\
\text { score } \\
\end{array}$ \\
\hline $12 \mathbf{a}$ & 5.959 & 6.07 & -0.111 & 2.95 & 6.07 & -0.111 & 2.95 \\
\hline $12 b$ & 6.149 & 6.35 & -0.201 & 2.95 & 6.33 & -0.181 & 2.95 \\
\hline $12 \mathrm{c}$ & 6.097 & 6.27 & -0.173 & 2.93 & 6.26 & -0.163 & 2.93 \\
\hline $12 d$ & 6.337 & 6.09 & 0.247 & 2.87 & 6.10 & 0.237 & 2.86 \\
\hline $12 \mathrm{e}$ & 6.187 & 5.81 & 0.377 & 2.87 & 5.89 & 0.297 & 2.87 \\
\hline $12 f$ & 5.920 & 6.01 & -0.090 & 2.85 & 5.99 & -0.070 & 2.85 \\
\hline $12 \mathrm{~g}$ & 5.678 & 6.00 & -0.322 & 2.81 & 6.11 & -0.432 & 2.80 \\
\hline $12 \mathrm{~h}$ & 6.319 & 6.32 & -0.001 & 2.89 & 6.36 & -0.041 & 2.89 \\
\hline $12 \mathrm{i}$ & 6.886 & 6.48 & 0.406 & 2.89 & 6.56 & 0.326 & 2.89 \\
\hline $12 \mathrm{j}$ & 5.796 & 6.12 & -0.324 & 2.87 & 6.02 & -0.224 & 2.87 \\
\hline $12 k$ & 5.921 & 6.34 & -0.419 & 2.85 & 6.32 & -0.339 & 2.85 \\
\hline 121 & 6.055 & 6.42 & -0.365 & 2.89 & 6.46 & -0.405 & 2.89 \\
\hline $12 \mathrm{~m}$ & 6.366 & 6.35 & 0.016 & 2.83 & 6.39 & -0.024 & 2.83 \\
\hline 120 & 6.252 & 6.36 & -0.108 & 2.76 & 6.48 & -0.228 & 2.63 \\
\hline $12 q$ & 5.268 & 5.37 & -0.102 & 2.81 & 5.32 & -0.052 & 2.81 \\
\hline $12 r$ & 5.222 & 5.08 & 0.142 & 2.82 & 5.27 & -0.048 & 2.82 \\
\hline $12 \mathrm{~s}$ & 6.125 & 6.04 & 0.085 & 2.85 & 6.00 & 0.125 & 2.85 \\
\hline $12 t$ & 5.854 & 5.85 & 0.004 & 2.82 & 5.90 & -0.046 & 2.82 \\
\hline $12 u$ & 6.602 & 6.43 & 0.172 & 2.77 & 6.64 & -0.038 & 2.77 \\
\hline $12 \mathrm{v}$ & 5.366 & 5.58 & -0.214 & 2.97 & 5.63 & -0.264 & 2.97 \\
\hline $12 w$ & 5.310 & 5.23 & 0.08 & 2.90 & 5.12 & 0.190 & 2.89 \\
\hline $12 x$ & 5.745 & 6.19 & -0.445 & 2.92 & 6.15 & -0.405 & 2.91 \\
\hline $12 y$ & 5.854 & 5.72 & 0.134 & 2.91 & 5.77 & 0.084 & 2.91 \\
\hline $12 z$ & 7.051 & 6.57 & 0.481 & 2.96 & 6.64 & 0.411 & 2.96 \\
\hline $12 \mathbf{a a}$ & 6.123 & 6.39 & -0.267 & 2.92 & 6.34 & -0.217 & 2.91 \\
\hline
\end{tabular}

